\title{
Des élèves bilingues en classe
}

1. Apprentissage des mathématiques

\section{Raúl Gagliardi}

\section{CpenEdition}

\section{Journals}

Édition électronique

URL : http://journals.openedition.org/ries/3965

DOI : 10.4000/ries.3965

ISSN : 2261-4265

Éditeur

Centre international d'études pédagogiques

Édition imprimée

Date de publication : 7 septembre 1995

Pagination : 87-94

ISSN : $1254-4590$

Référence électronique

Raúl Gagliardi, «Des élèves bilingues en classe », Revue internationale d'éducation de Sèvres [En ligne], 07 | 1995, mis en ligne le 02 octobre 2014, consulté le 30 avril 2019. URL : http:// journals.openedition.org/ries/3965; DOI : 10.4000/ries.3965

Ce document a été généré automatiquement le 30 avril 2019

(c) Tous droits réservés 


\title{
Des élèves bilingues en classe
}

\author{
1. Apprentissage des mathématiques
}

\section{Raúl Gagliardi}

1 Le Bureau international d'éducation - UNESCO poursuit le développement de son projet sur l'éducation interculturelle/multiculturelle et la formation des enseignants. L'analyse que nous présentons ici est une synthèse de certains résultats obtenus au cours de la première année.

2 Nous voulons discuter ici de certains problèmes d'apprentissage des mathématiques que rencontrent les enfants qui ne parlent pas la langue d'enseignement, des enfants «bilingues ». Nous avons choisi les mathématiques en raison de l'importance qu'elles ont dans la réussite scolaire et sociale. Autre élément que nous avons considéré : l'existence de bonnes études sur les relations entre langage, culture et apprentissage des mathématiques.

3 Nous croyons que la plupart des indications qui sont suggérées dans les études indiquées peuvent être appliquées aux problèmes d'apprentissage des autres disciplines, en particulier des sciences.

\section{Développement du langage et apprentissage des mathématiques}

4 Le bilinguisme a été fréquemment considéré comme un handicap pour l'école. Cependant, différentes recherches sur l'apprentissage des mathématiques montrent que des élèves ayant une bonne connaissance d'une langue n'ont pas de problèmes d'apprentissage particuliers. Ceux qui ont une bonne connaissance de deux langues ont en général des avantages par rapport aux monolingues. Par contre, les élèves qui n'ont pas suffisamment d'acquis en langue, ont des problèmes d'apprentissage pour les mathématiques.

5 Une question importante se pose. Pourquoi un faible développement du langage est-il un obstacle pour l'apprentissage des mathématiques ? Certains éléments de réponse peuvent être obtenus à partir de recherches élaborées sur les difficultés d'apprentissage des élèves 
d'origine finnoise en Suède, qui ont démontré que des élèves qui ne parlaient pas bien le suédois n'avaient pas de problèmes d'apprentissage des mathématiques malgré des cours donnés en suédois. Le bon développement du finnois était fondamental pour apprendre les mathématiques bien que ces dernières fussent enseignées en suédois. À partir de ces résultats on peut faire l'hypothèse que le bon développement de la langue maternelle est un réquisit pour l'apprentissage des mathématiques, même si elles sont enseignées dans une autre langue. Cette hypothèse est considérée comme tellement importante par certains auteurs qu'ils postulent que « tout cours de mathématique est d'abord un cours de langue ».

6 Selon Mac Gregor ${ }^{1}$, le langage a un rôle important dans l'organisation de la connaissance et dans la pensée logique. L'apprentissage des mathématiques est lié aux explications orales et écrites. Fréquemment, les concepts mathématiques sont définis par des relations entre objets. Ces relations ne peuvent pas être touchées ou signalées par le doigt. Pour cette raison, l'apprentissage des mathématiques a besoin d'un bon niveau de langage. La verbalisation est essentielle pour l'abstraction, la généralisation et la classification en catégories. Le langage oral est aussi nécessaire pour la présentation des résultats en classe. L'élève qui n'a pas bien assimilé son langage a de grandes difficultés pour exprimer en mots les relations entre les objets, ce qui provoque des difficultés pour la construction des concepts mathématiques.

7 Il existe un autre problème lié à l'apprentissage des mathématiques: la nécessité de construire de nouveaux concepts et de les définir avec des mots précis. Un faible développement du langage peut être un obstacle pour construire ces réseaux conceptuels.

La notation mathématique n'est pas une version simplifiée du langage naturel. Au contraire, la syntaxe des mathématiques est différente de celle de l'anglais, ou de n'importe quelle autre langue naturelle. L'apprentissage de la notation mathématique non seulement est utile en classe mais il permet le développement d'un niveau supérieur d'abstraction et d'organisation des activités.

9 Il a été démontré que la capacité de développer la notation mathématique est en relation avec la langue que parle l'élève. Dans une recherche effectuée aux États-Unis d'Amérique, Orr ${ }^{2}$ étudie l'apprentissage des mathématiques par des étudiants avancés qui parlent l'anglais des afro-américains (Black English Vernacular). Il démontre que beaucoup de difficultés de l'apprentissage des mathématiques sont provoquées par les différences entre la syntaxe de cet anglais et celle de l'anglais technique (Standard Technical English). Par exemple, des erreurs dans la distance et la direction peuvent être en rapport avec des expressions de l'anglais des afro-américains qui ne fait pas la différence entre la distance et la localisation.

\section{Apprentissage des mathématiques}

\section{Carence de concepts mathématiques dans la langue et la culture}

L'apprentissage des sciences et des mathématiques a besoin du développement du langage et d'une "vision du monde» cohérente avec la mathématique et la science. L'enfant ne développe pas seulement un langage mais une capacité pour percevoir certaines données et les structurer dans des réseaux de plus en plus complexes. En d'autres termes, le problème du bilinguisme ne doit pas être réduit aux seules difficultés 
du langage. Il faut considérer le type de pensée et la vision du monde qui sont liés au langage et à la culture.

11 Un exemple de cette situation est celui des élèves aborigènes en Australie, qui ont été éduqués dans des communautés aborigènes traditionnelles. Fréquemment, ces élèves apprennent par cœur les opérations arithmétiques sans les comprendre réellement. Ils ont souvent des difficultés quand on leur demande d'expliquer en anglais standard ce qu'ils ont fait.

12 Les difficultés d'apprentissage des mathématiques ont été analysées par Kepert ${ }^{3}$ qui a étudié les relations entre la langue, la capacité de communication en mathématique et l'apprentissage des mathématiques. Des sujets tels que les nombres, les formes en deux ou trois dimensions ou la probabilité sont facilement discutés par des élèves de l'école élémentaire australienne qui parlent l'anglais comme langue maternelle. Au contraire, les enfants aborigènes souvent n'ont pas le langage mathématique ni la perspective culturelle pouvant reconnaitre ces concepts. Les communautés aborigènes traditionnelles de l'Australie n'ont pas le concept de numéro. Elles ont une organisation sociale fondée sur les réseaux familiers qui n'ont aucun élément de mesure numérique. Le concept même du chiffre n'existe pas. Les langues aborigènes n'ont pas de mots pour les chiffres. L'élève qui n'a ni dans sa culture, ni dans sa langue maternelle, le concept du nombre se voit confronté à l'école aux difficultés de l'apprentissage des mathématiques, fondé sur une langue et une culture dominantes qui contient comme élément de base fondamental le nombre et comme activité fondamentale la mesure numérique. Par contre, l'élève dont l'anglais est la langue maternelle, a assimilé de ce fait toute une série de concepts mathématiques de base; il a intégré la mesure et le nombre dans sa pensée et son langage. Quant aux enfants qui ont eu une autre éducation, comme l'aborigène australien, eux ont développé une autre vision du monde.

13 Selon Christie $^{4}$, la vision du monde d'une population souligne tous les aspects de sa vie religion, langage, système social - y compris sa façon d'organiser les activités journalières. La vision du monde n'a pas un développement spontané. Elle est développée en fonction des objectifs culturels et des modèles de relations sociales. Elle est aussi développée en fonction des contraintes environnementales. Cependant, si la vision du monde est le résultat d'un long développement, c'est aussi un facteur important pour le développement.

14 Les caractéristiques de la vision du monde aborigène ont déterminé que les notions de quantité - plus ou moins, nombres, mathématiques et pensée positive - ne sont pas importantes pour les aborigènes. Quand ils regardent le monde, ils se concentrent sur les qualités et les relations qui sont apparentes, les quantités n'étant pas pertinentes. Une vision du monde dans laquelle la terre, les êtres spirituels, les personnes et les arbres sont unifiés, ne permet pas une analyse scientifique. Cependant, la vision du monde des aborigènes permet d'établir des relations cohérentes entre la population, la nature, la terre et le temps. Dans un monde dans lequel les mathématiques et la science n'ont pas de place, les unités spirituelles que transcende l'analyse occidentale continuent d'exister.

15 La communication des mathématiques n'est pas une tâche facile dans beaucoup d'écoles aborigènes australiennes. Les connaissances traditionnelles, la culture et la structure sociale des communautés aborigènes peuvent être utilisées dans les cours de mathématiques (Kepert, 1993). Par exemple, le système complexe des relations familiales, caractéristique des communautés aborigènes, ou la connaissance qu'ils ont du territoire, 
peuvent être la base du développement de la capacité d'abstraction, nécessaire en mathématique.

En d'autres termes, l'enfant qui n'a pas été éduqué dans une certaine vision du monde et dans un langage qui contient les éléments essentiels pour la quantification, a de grandes difficultés pour apprendre les mathématiques. Cependant, il a développé d'autres capacités, telles que la capacité de comprendre des réseaux de famille ou d'utiliser les ressources du territoire. Ces capacités peuvent être la base d'un nouveau modèle d'enseignement des mathématiques et des sciences.

17 Fréquemment, les enseignants australiens non-aborigènes ont de grandes difficultés à utiliser la culture et les connaissances des élèves aborigènes. Dans certaines expériences, des enseignants aborigènes ont aidé d'autres enseignants à mieux comprendre la culture traditionnelle. L'apprentissage des éléments d'une culture différente est en soi un excellent moyen de formation des enseignants. Cela peut les aider à sortir du «cocon culturel » et les amener à mieux comprendre les autres cultures. Cette proposition n'est pas limitée aux enseignants de mathématiques.

Un autre problème fréquent chez les élèves aborigènes est lié au fait qu'ils parlent un anglais aborigène différent de l'anglais standard. Ces variations, si cet anglais est parlé rapidement, sont incompréhensibles pour ceux qui ne le parlent pas. Comme dans le cas de l'anglais afro-américain des États-Unis d'Amérique, les similitudes apparentes entre l'anglais standard et les autres formes peuvent générer des confusions au niveau de l'école. Des linguistes australiens sont en train de démontrer que l'anglais aborigène doit être considéré comme une langue différente et non comme une variation de l'anglais standard (Christie 4).

19 L'exemple des difficultés des élèves aborigènes australiens nous montre l'importance de prendre en considération le langage et la culture des élèves des autres communautés et de ne pas penser a priori qu'ils ont les mêmes modèles de pensée que les élèves de la culture dominante.

\section{L'expérience de la Nouvelle-Zélande}

\section{L'enseignement des mathématiques et des sciences en maori}

La division du développement des programmes d'étude du département d'éducation de la Nouvelle-Zélande est en train de chercher de nouvelles stratégies pour améliorer l'enseignement des mathématiques destiné aux étudiants maoris (Beeg5). Ces stratégies sont fondées sur trois éléments: langage, culture et confiance en soi (self-esteem). Les questions auxquelles il faut répondre, sont :

- doit-on enseigner les mathématiques comme un autre cours, et simplement l'enseigner en maori?

- l'enseignement des mathématiques doit-il commencer par les connaissances mathématiques traditionnelles des Maoris?

- l'enseignement des mathématiques doit-il suivre l'approche holistique, caractéristique de l'éducation traditionnelle maorie?

21 La réponse à ces questions est centrée sur un élément important. Il ne s'agit pas seulement de donner des cours en maori, mais d'intégrer, dans le contenu, les 
applications et les méthodes d'enseignement, les caractéristiques d'une société biculturelle comme la société de la Nouvelle-Zélande.

Les méthodes d'enseignement utilisées dans les cours de mathématiques dans les écoles supérieures peuvent être classées en quatre grandes catégories.

- Enseignement dans le style occidental. C'est le plus fréquent; il est utilisé pour les élèves non maoris et pour les élèves maoris. Il est basé sur les besoins et les expériences occidentales. Le développement de la confiance en soi des élèves n'est pas pris en considération et les cours sont axés sur le contenu.

- Enseignement biculturel. Dans un style similaire à l'occidental, il prend soin d'intégrer la culture et les règles de communication maories (par exemple, on accepte le fait que regarder vers le bas est un symbole de respect au lieu de regarder directement en face). Quelques applications mathématiques se font dans un contexte maori (par exemple des leçons sur la mesure se font en utilisant la structure traditionnelle de la maison maorie). L'enseignant intègre des exemples provenant du langage, de la culture ou des mathématiques maories (par exemple pour analyser les transformations, il étudie des dessins traditionnels maoris).

- Enseignement bilingue. Il est supposé que les caractéristiques biculturelles sont incorporées et que l'enseignement se fait en maori quand cela est possible. Pour cette raison ce style d'enseignement a besoin de traduire en maori les concepts mathématiques. Une question importante à résoudre est de savoir s'il est préférable d'enseigner les mathématiques occidentales en maori ou s'il est plus important de mieux connaitre les mathématiques traditionnelles maories et les utiliser comme point de départ.

- Enseignement holistique. Une des caractéristiques de l'éducation traditionnelle maorie est qu'elle forme un tout, c'est-à-dire que lors d'un cours toutes les disciplines sont abordées. Elles sont intégrées à l'enseignement. Par contre, une approche holistique est utilisée. Cette approche signifie que certains thèmes sont transversaux. Le glossaire (syllabus) maori des mathématiques a quinze thèmes communs à tous les cours : la généalogie, l'école, le corps, la récréation, la ville, la nourriture, le lieu d'origine, les événements, le deuil, le travail, les légendes, le monde changeant, les aspirations et les personnages célèbres.

Un autre élément important de l'enseignement des mathématiques est celui de l'évaluation. Quelques évidences indiquent que les élèves maoris ne veulent pas avoir d'appréciations personnelles ou être considérés meilleurs ou pires que les autres. Ils insistent sur l'importance du travail en groupe et sur la nécessité de travailler plus souvent de cette façon. Autre caractéristique des élèves maoris : ils n'ont pas l'habitude de faire des critiques envers des personnes d'autorité. Par conséquent, il est difficile de les faire participer à une discussion sur les enseignants ou sur ce que les enseignants demandent. La culture maorie est une culture orale et il faut aussi vérifier le style d'enseignement avec lequel les élèves maoris sont plus à l'aise.

Cet exemple montre comment des facteurs culturels peuvent jouer un rôle très important dans la réussite des élèves.

Autre facteur qui est devenu très important en Nouvelle-Zélande : la volonté des Maoris de diffuser leur langue qui était en train de disparaître. La diffusion de la langue maorie a été liée à celle de la culture maorie et à l'existence des écoles maories. Le processus a commencé par la création d'écoles préscolaires en langue maorie, organisées selon le modèle traditionnel maori dans lequel des enfants d'âge différent peuvent coexister dans un même groupe. Les plus âgés participent activement aux soins et à l'éducation des plus petits. 

particulières lors de l'apprentissage des mathématiques. Ceux qui ont bien assimilé leur langue maternelle mais ne parlent pas bien la langue d'enseignement, peuvent avoir aussi des difficultés d'apprentissage des mathématiques. Dans les deux cas, les enseignants jouent un rôle important. L'élément fondamental de l'aide qu'ils peuvent apporter est l'acceptation du fait que les problèmes des élèves ne sont pas un handicap permanent, que ces élèves ne sont pas moins intelligents ou capables que les élèves qui maîtrisent la langue d'enseignement. Les enseignants et les autres acteurs du système éducatif prennent conscience qu'il faut éliminer les stéréotypes négatifs vis-à-vis des enfants d'une autre culture. 


\section{NOTES}

1. M. Mac Gregor, «Language, culture and mathematics learning ", dans Teaching Mathematics in the Multicultural Classroom: a resource for teachers and teachers educators, édité par Mac Gregor, M. et Moore, R., School of Science and Mathematics Education, Institute of Education, The University of Melbourne, Australie, 1991, p. 6-20.

2. E.W. Orr (1987), « Twice as less ", New York: Norton. Mathematical Behaviour, 11, 261. Cité dans Mac Gregor, 1993.

3. B. Kepert, "Aboriginal Students Communicating Mathematics", dans Communicating Mathematics: perspectives from classroom practice and current research, edited by $\mathrm{M}$. Sthephens, A. Waywood, D. Clarke et J . Izard; The Australian Council for Educational Research Ltd., Victoria, Australie, 1993, p. 274-282.

4. M.J. Christie, Aboriginal perspectives on Experience and Learning: The Role of Language in Aboriginal Education, Deakin University, Victoria, Australie, 1992.

5. A. Begg, «Mathematics, Maori Language and Culture », dans Modelling, Application and Applied Problem Solving, édité par Blum, Niss et Huntley, Ellis Howard Ltd., Chichester (chapitre 8), 1989.

\section{INDEX}

Index géographique : Australie, Nouvelle-Zélande, États-Unis

Mots-clés : enseignement bilingue, enseignement des mathématiques, langue d'enseignement, langue d'origine, discipline non linguistique

\section{AUTEUR}

\section{RAÚL GAGLIARDI}

Conseiller technique du projet sur la formation des enseignants et l'éducation multiculturelle/ interculturelle, BIE-UNESCO, Genève, Suisse 